\title{
Efeito do genótipo e da idade de ovinos na reatividade medida em pista de venda
}

\author{
Isabella Dias Barbosa Silveira ${ }^{1}$, Vivian Fischer ${ }^{2}$, Gilson de Mendonça $^{3}$ \\ 1 Universidade Federal de Pelotas - UFPel, Departamento de Zootecnia, Caixa postal 354, Campus Universitário - Pelotas, RS, CEP: $96010-970$. \\ 2 Universidade Federal do Rio Grande do SUI-UFRGS. \\ ${ }^{3}$ Universidade Federal do Pampa - UNIPAMPA
}

RESUMO - Objetivou-se avaliar o comportamento de ovinos e relacionar aspectos comportamentais com a raça e idade dos animais. Foram observados 5.971 animais divididos em 181 lotes quanto às raças - Corriedale $(\mathrm{n}=2.731)$, Ideal $(\mathrm{n}=1.574)$, Merino $(\mathrm{n}=168)$, Texel $(\mathrm{n}=717)$, Suffolk $(\mathrm{n}=247)$ e 6 mestiços $(\mathrm{n}=534)$ - e quanto à idade - cordeiras $(1,5$ ano, $n=3.021)$, ovelhas (acima de 1,5 ano, $\mathrm{n}=2457$ ) e cordeiras dente-de-leite (média de 6 meses de idade, $\mathrm{n}=493$ ). $\mathrm{O}$ comportamento médio dos lotes foi avaliado pela atribuição de escores de movimentação e resistência à condução na entrada, permanência e saída da pista de comercialização. Os animais das raças mais especializadas para produção de carne (Suffolk e Texel) e os animais jovens foram os mais agitados e apresentaram maior resistência à condução pelo homem em comparação àqueles das raças produtoras de lã e aos mais velhos. Os escores comportamentais foram positivamente relacionados entre si, o que permite a escolha de qualquer um deles para medir o comportamento dos ovinos. O comportamento de ovinos em pista de venda é influenciado pela idade e raça.

Palavras-chave: agitação, comportamento, escores, raças, temperamento

\section{Effects of genotype and age of sheep in reactivity measured at market auction}

\begin{abstract}
The objective of this work was to evaluate behavior of sheep and to relate behavioral aspects with breed and age of animals. It was observed 5,971 animals distributed in 181 plots regarded to: breed - Corriedale ( $\mathrm{n}=2,731$ ), Ideal $(n=1,574)$, Merino $(n=168)$, Texel $(n=717)$, Suffolk $(n=247)$ and six crossbreds $(n=534)$; age - yearlings $(1.5$ year, $n=3,021)$, ewes (above 1.5 year, $n=2,457$ ) and lambs (average 6 months of age, $n=493$ ). Average behavior of plots was evaluated by attributing scores of movement and resistance to conduction at entrance, permanence and exit from the market auction. Animals from more specialized breeds for meat production (Sulfock and Texel) and young animals were the most agitated and they showed more resistance to human conduction compared to those wool producer breeds and to the older ones. Behavior scores positively correlated among each other, allowing to choose any of them to measure sheep behavior. Sheep on market auction behavior is influenced by age and breed.
\end{abstract}

Key Words: agitation, behavior, breeds, scores, temperament

\section{Introdução}

Uma das características mais notórias no comportamento de cabras e ovelhas é a coesão e o agrupamento social (Syme \& Elphick, 1983), comportamento básico e útil na transmissão de aprendizagem e que permite a organização de um número de animais em unidades familiares, grupos de acasalamento, alimentação, grupos de pastejo, entre outros. O temperamento deve ser considerado no manejo na fazenda, no transporte e no abatedouro, pois expressa a reação dos animais nessas situações.

O temperamento pode ser expresso pelas reações dos animais em relação ao homem, geralmente atribuídas ao medo, o que possibilita avaliá-lo por meio de modificações comportamentais ou fisiológicas (Fordyce et al., 1982; 1988). A reatividade dos animais é inata, mas pode ser modificada pela experiência prévia (Grandin \& Deesing, 1998), especialmente nos primeiros meses de vida do animal ou até o início da fase pós-desmama (Boivin et al., 1992).

Situações que provocam o estresse crônico nos animais reduzem seu bem-estar e podem prejudicar o sistema imune (Blecha et al., 1984), a eficiência reprodutiva (Hixon et al., 1981; Stermer et al., 1981; Stobel \& Moberg, 1982) e o desempenho produtivo, como ganho de peso (Voisinet et al., 1997; Barbosa Silveira et al., 2006), portanto, o 
temperamento trata-se de uma característica de interesse econômico (Paranhos da Costa, 2001).

Além das diferenças individuais, genótipos distintos reagem diferentemente ao manejo (Shupe, 1978; Whatley et al., 1974), por exemplo, ovinos da raça Rambouillet tendem a manter-se em grupo, enquanto os da raça Cheviots são mais independentes.

Em ovinos o campo visual varia de 191 a $306^{\circ}$, variação relacionada à quantidade de lã na cabeça (Grandin, 1989). Observações durante a venda de bovinos em pistas de remate indicam que esses animais tendem a se assustar em resposta a estímulos súbitos e intermitentes (Lanier et al., 2000). O temperamento agitado do animal pode se constituir em risco para si próprio, para os outros animais e para o homem (Fordyce et al., 1988; Grandin, 1993).

Os ovinos são animais gregários e, por sua natureza, relutam à separação e à mistura a outros animais. Quando são apresentados subitamente a novas situações, reagem com estresse e podem resistir às atividades de condução (Grandin, 2000). O objetivo neste trabalho foi avaliar a influência da raça e idade de ovinos no temperamento em pista de remate.

\section{Material e Métodos}

Foram utilizados dados de observações de 5.971 animais divididos em 181 lotes, com observações em dois anos consecutivos (2003-2004), durante a realização das XIX e XX edições da FEOVELHA, em Pinheiro Machado, Rio Grande do Sul.

Os animais foram identificados quanto à raça em Corriedale $(n=2.731)$, Ideal $(n=1.574)$, Merino $(n=168)$, Texel ( $(n=717)$, Suffolk $(n=247)$ e mestiços $(n=534)$ e quanto à idade em cordeiras $(1,5$ ano, $n=3.021)$, ovelhas (acima de 1,5 ano, $\mathrm{n}=2.457$ ) e cordeiras dente-de-leite (média de 6 meses de idade, $n=493$ ).

A pista de remate é coberta e com piso de areia. Os animais estavam em jejum de aproximadamente 12 horas e os dias de coleta estavam ensolarados com temperatura em torno de $35^{\circ} \mathrm{C}$ e ausência de ventos.

Os dois observadores foram previamente treinados em outros experimentos e foram os mesmos nos dois anos de avaliação; localizaram-se na quinta fileira de assentos, de onde podiam observar a entrada, permanência e saída dos animais da pista, sem interferir em sua movimentação e zona de fuga.

O temperamento foi avaliado por meio da atribuição de escores comportamentais conforme a agitação dos animais e pela consequente facilidade para realizar o manejo em pista, adaptado de Piovesan (1998). As avaliações foram realizadas em três momentos: entrada na pista; movimentação na pista; e saída da pista. Na entrada na pista, foram observados quanto à maneira como reagiram à condução para entrada na pista de remate e classificados em escores de 1 a 3 , em que: 1 = entra na pista sem ajuda no momento em que é aberta a porteira; 2 = precisa ser conduzido para a pista com a utilização de guizos ou similares após a abertura da porteira; 3 = resiste mais de uma vez à entrada na pista e precisa ser conduzido com a utilização de guizos ou similares. Animais com maior resistência receberam valores de escore mais elevados e foram classificados como mais temerosos ou resistentes à entrada em pista. $\mathrm{Na}$ avaliação do temperamento em movimentação na pista, os animais foram classificados de 1 a 4 de acordo com o grau de perturbação em relação à interferência humana e à emissão de sons do leiloeiro e do público: 1 = caminha, quieto; 2 = caminha, trota continuamente; 3 = mais veloz que o trote; e 4 = golpe de cabeça trota constantemente. Na classificação do tempera-mento na saída de pista, os animais receberam os seguintes escores: 1 = sem ajuda; 2 = com ajuda; 3 = resistência, de modo que aqueles com maior resistência receberam valores de escore mais elevados e foram classificados como mais temerosos ou resistentes à saída da pista. Os escores foram atribuídos aos lotes de animais com base no apresentado pela maioria dos animais do lote. Foram registrados a raça, o número de animais por lote, o número do lote e a idade dos animais.

Os dados foram analisados pelo SAS, versão 6.12, procedimentos FREQ e CORR. As médias dos escores de entrada na pista, movimentação na pista e saída da pista de venda, de natureza discreta, foram submetidas à análise não-paramétrica pelo teste Cochran-Mantel-Haenzel para verificar a associação global entre as raças e idades e as variáveis dependentes. Na análise de correlação (Spearman), as raças foram classificadas de acordo com sua aptidão, da mais laneira para a mais especializada em carne: 1 = Merino, 2 = Ideal, 3 = Corriedale, $4=$ mestiça, $5=$ Texel $\mathrm{e}$ 6 = Suffolk. As idades foram classificadas como: $1=$ cordeira dente-de-leite, 2 = cordeira e 3 = ovelha. $\mathrm{Na}$ análise não-paramétrica, os animais foram classificados conforme o escore para cada variável comportamental. Animais com escore 1 foram classificados como nãoreativos e aqueles com o maior escore foram classificados como reativos, enquanto os demais foram considerados intermediários. O nível de mínimo de significância para rejeição da hipótese de nulidade foi de 0,10. 


\section{Resultados e Discussão}

Segundo a análise descritiva, os valores de média, moda e mediana do número de animais por lote foram, respectivamente, 33, 25 e 25, com mínimo e máximo de 10 e 250 animais/lote. O número de animais avaliados no primeiro e segundo ano foi de 3.341 e 2.630 e o número de lotes por raça e em cada ano, de 3 e 3 (Merino), 10 e 34 (Ideal), 39 e 33 (Corriedale), 11 e 12 (mestiças), 15 e 14 (Texel) e 3 e 1 (Suffolk). O número de lotes por idade e em cada ano foi de: cordeiras dente-de-leite (0 e 19); cordeiras (43 e 34); ovelhas (41 e 44).

No primeiro ano de avaliação, a frequência de animais classificados como reativos foi significativa e menor em relação ao segundo ano de acordo com os escores de entrada e movimentação na pista, porém não houve diferença significativa no escore de saída da pista de venda (Tabela 1).

Embora indiquem a agitação ou movimentação dos animais, as variáveis comportamentais foram medidas em momentos distintos, o que pode ter provocado respostas diferentes por parte dos animais. Os animais tendem a resistir mais à entrada a ambientes novos, sobretudo se forem movimentados de ambientes mais claros para outros mais escuros (Grandin \& Dessing, 1998; Grandin, 2000). Além disso, se assustam com barulhos agudos e intermitentes (Lanier et al., 2000), o que efetivamente ocorreu com a saída dos animais dos currais de espera para a pista de remate e, depois, durante o leilão. Possivelmente essas duas situações provocam maior sensação de medo, sobretudo naqueles menos habituados à manipulação, ou seja, as cordeiras dentes-de-leite. Desse modo, a diferença na proporção de animais reativos entre os anos de avaliação foi atribuída, parcialmente, à presença dessa categoria mais jovem

O percentual de animais reativos, ou seja, com maiores escores, diminuiu conforme aumentou a idade e provavelmente foi modulado pela experiência prévia, pois, à medida que aumenta a idade, normalmente tornam-se mais frequentes o manejo e contato pelo homem (Tabela 2).
Esses resultados estão de acordo com relatos de Grandin (1993) de que animais mais jovens sofrem mais os efeitos dos agentes estressores, devido à menor experiência prévia. Situações novas, como uma pista de remates, tem forte efeito estressante nos animais (Grandin \& Deesing, 1998). A experiência prévia de cada tem efeito importante sobre a magnitude da resposta de medo (Hemsworth \& Barnett, 1991).

Os animais das raças Suffolk e Texel, de maior aptidão zootécnica para produção de carne, seguidos dos mestiços, apresentaram os maiores percentuais de reatividade, segundo os três escores de movimentação (Tabela 3).

Os processos de medo e ansiedade, em parte, estão relacionados ao controle genético, que pode contribuir para a existência de diferenças individuais (Bouchard et al., 1990). Em várias espécies domésticas, o componente genético determina parte das diferenças individuais na resposta ao contato do homem. A herdabilidade da reação de defesa ao homem tem mostrado valores consistentes em bovinos leiteiros (Dickson et al., 1970), bovinos de carne (Le Neindre et al., 1995) e suínos (Hemswort et al., 1990). O mesmo acontece nas populações de raças ovinas, como observado para as raças Romanov e Ile de France (Romeyer \& Bouissou, 1992)

Diferenças genéticas podem afetar as reações de estresse durante o manejo, de forma que animais que possuem genética excitável são mais propensos a se agitar quando confrontados a uma situação nova. A seleção unilateral para características produtivas, como rápido ganho de peso ou musculatura magra determinou em suínos e bovinos temperamento mais excitável (Grandin, 1993). Em comparação às linhagens genéticas mais antigas, que possuíam mais gordura, observações indicam que os híbridos magros são mais excitáveis e difíceis de manejar.

Suínos híbridos magros possuem tendência mais acentuada a se assustarem, daí a maior dificuldade de separar um animal do grupo. Esses animais apresentam comportamentos característicos, como alta reatividade ao toque, extremo instinto gregário, constante retorno na fila durante o manejo pré-abate e alta aversão ao gás carbônico

Tabela 1 - Frequência absoluta e relativa (\%) de reatividade de ovinos em pista de venda em dois anos de avaliação

\begin{tabular}{|c|c|c|c|c|c|c|c|}
\hline & \multicolumn{3}{|c|}{ Ano 1} & \multicolumn{3}{|c|}{ Ano 2} & \multirow[b]{2}{*}{$\mathrm{P}>\mathrm{CMH}$} \\
\hline & Não-reativos & Intermediários & Reativos & Não-reativos & Intermediários & Reativos & \\
\hline Escore de entrada na pista & $76(90,5)$ & $7(8,3)$ & $1(1,2)$ & $70(72,1)$ & $12(12,4)$ & $15(15,5)$ & 0,0004 \\
\hline Escore de movimentação na pista & $46(54,5)$ & $38(45,5)$ & $0(0)$ & $63(65)$ & $25(25,8)$ & $9(9,2)$ & 0,0677 \\
\hline Escore de saída da pista & $62(73,8)$ & $20(23,8)$ & $2(2,4)$ & $74(76,3)$ & $16(16,5)$ & $7(7,2)$ & 0,7766 \\
\hline
\end{tabular}

CMH teste de correlação linear de Cochran-Mantel-Haenzel.

Valores de frequência relativa (\%) apresentados dentro dos parênteses. 
Tabela 2 - Frequência absoluta e relativa (\%) da reatividade de ovinos em pista de venda em dois anos de avaliação

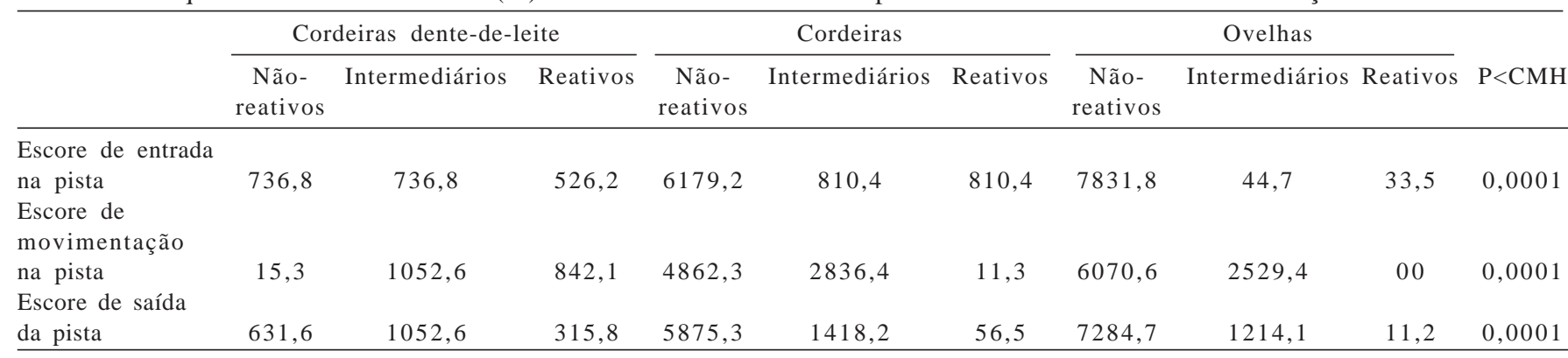

$\mathrm{CMH}=$ teste de correlação linear de Cochran-Mantel-Haenzel

Valores de frequência relativa apresentados na linha inferior.

(Grandin, 1992). Essas investigações comprovam que seria vantajoso selecionar com a finalidade de excluir animais de temperamento muito excitável (Grandin, 2000).

Observaram-se correlações significativas entre as variáveis comportamentais, sobretudo os coeficientes de correlação médios e positivos entre os escores de entrada na pista, movimentação na pista e de saída da pista, demonstrando associação entre eles (Tabela 4). Resultados semelhantes são descritos para bovinos por Curley et al. (2004) e Silveira Barbosa et al. (2008). Também foram observadas correlações negativas entre a idade e os escores comportamentais, corroborando os resultados previamente descritos, de que, em animais mais jovens a frequência de classificação em intermediários e reativos é bem maior. Detectou-se correlação positiva entre a ordenação das raças (classificadas da mais laneira até as mais especializadas na

Tabela 3 - Frequência absoluta e relativa (\%) da reatividade de ovinos em pista de venda em dois anos de avaliação

\begin{tabular}{|c|c|c|c|c|}
\hline Raça & Reatividade & $\begin{array}{l}\text { Escore de entrada } \\
\text { da pista }\end{array}$ & $\begin{array}{c}\text { Escore de movimentação } \\
\text { na pista }\end{array}$ & $\begin{array}{l}\text { Escore de saída } \\
\text { da pista }\end{array}$ \\
\hline \multirow[t]{2}{*}{ Merino } & Não-reativos & $66(91,7)$ & $51(70,8)$ & $64(88,9)$ \\
\hline & Intermediários & $4(5,6)$ & $21(28,2)$ & $6(8,3)$ \\
\hline \multirow[t]{3}{*}{ Ideal } & Não-reativos & $37(84,1)$ & $39(88,6)$ & $42(95,5)$ \\
\hline & Intermediários & $3(6,8)$ & $4(9,1)$ & $2(4,6)$ \\
\hline & Reativos & $4(9,1)$ & $1(2,3)$ & $0(0)$ \\
\hline Corriedale & Reativos & $1(11,1)$ & $1(11,1)$ & $0(0)$ \\
\hline \multirow[t]{3}{*}{ Mestiços } & Não-reativos & $19(65,5)$ & $3(10,3)$ & $11(37,9)$ \\
\hline & Intermediários & $4(13,8)$ & $23(79,3)$ & $14(48,3)$ \\
\hline & Reativos & $6(20,7)$ & $3(10,3)$ & $4(13,8)$ \\
\hline \multirow[t]{2}{*}{ Texel } & Não-reativos & $2(50)$ & $0(0)$ & 1 (25) \\
\hline & Intermediários & 1 (25) & $4(100)$ & $2(50)$ \\
\hline $\mathrm{P}<\mathrm{CMH}$ & & 0,009 & 0,0001 & 0,0001 \\
\hline
\end{tabular}

$\mathrm{CMH}=$ teste de correlação linear de Cochran-Mantel-Haenzel.

Valores de frequência relativa (\%) apresentados dentro dos parênteses.

Tabela 4 - Valores dos coeficientes de correlação de Spearman entre as variáveis comportamentais descritoras do temperamento, raças e idades de ovinos avaliados em pista de remate

\begin{tabular}{|c|c|c|c|c|c|}
\hline & $\begin{array}{c}\text { Escore de entrada } \\
\text { na pista }\end{array}$ & $\begin{array}{c}\text { Escore de movimentação } \\
\text { na pista }\end{array}$ & $\begin{array}{c}\text { Escore de saída } \\
\text { da pista }\end{array}$ & Raça & Idade \\
\hline Escore de entrada na pista & - & $0.42^{* * *}$ & $0.52^{* * *}$ & $0.28^{* *}$ & $-0.34^{* *}$ \\
\hline Escore de movimentação na pista & $0.42^{* * *}$ & - & $0.54^{* * *}$ & $0.40^{* * *}$ & $-0.38^{* * *}$ \\
\hline Escore de saída da pista & $0.52^{* *}$ & $0.5^{* * *}$ & - & $0.41^{* * *}$ & $-029^{* * *}$ \\
\hline Raça & $0.28^{* *}$ & $0.40^{* * *}$ & $0.4^{* * *}$ & & 22 \\
\hline Idade & $-0.34^{* *}$ & $-0.38^{* * *}$ & $0,29^{* * *}$ & $-0,22^{* *}$ & - \\
\hline
\end{tabular}

${ }^{* * *} \mathrm{P}<0,0001 ;{ }^{* *} \mathrm{P}<0,05 ; * \mathrm{P}<0.10$. 
produção de carne), o que indica que os animais das raças com maior aptidão cárnea apresentaram maiores valores dos escores comportamentais.

Outros estudos com ovinos relacionaram maior agitação com temperamento excitável. Diáz (2007) verificou que os ovinos mais estressados apresentaram maior movimentação no teste de arena e que os ovinos que se agitaram mais durante o teste de arena e o teste de tempo de fuga no brete apresentaram maiores valores de temperatura corporal e frequência cardiorrespiratória durante o teste de contenção, o que, segundo Fraser \& Broom (1997), é sintomático do estresse.

Rech et al. (2008) observaram que ovelhas mais agitadas durante a identificação dos seus cordeiros, feita até 24 horas após o parto, se isolaram menos do rebanho, protegeram menos seus cordeiros e amamentaram por menos tempo, desmamando-os mais leves.

\section{Conclusões}

A raça e a idade influenciam o temperamento de ovinos em pista de remate, o que pode servir para predizer quais animais serão mais propensos ao estresse ou que apresentarão maior movimentação e agitação quando expostos a novas situações, como um confinamento ou uma planta frigorífica.

\section{Referências}

BARBOSA SILVEIRA, I.D.; FISCHER, V.; SOARES, G.J.D. Relação entre o genótipo e o temperamento de novilhos em pastejo e seu efeito na qualidade da carne. Revista Brasileira de Zootecnia, v.35, n.2, p.519-526, 2006.

BARBOSA SILVEIRA, I.D.; FISCHER, V.; WIEGAND, M.M. Temperamento de bovinos de corte: métodos de medida em diferentes sistemas produtivos. Archivos de Zootecnia, v.57, p.321-332, 2008.

BLECHA, F.; BOYLES, S.L.; RILEY, J.G. Shipping suppresses lymphocyte blastogenic responses in Angus and Bhahman $\mathrm{x}$ Angus feeder calves. Journal of Animal Science, v.59, p.576-583, 1984.

BOIVIN, X.; LE NEINDRE, P.; CHUPIN, J.M. Establisment of cattle-human relationship. Applied Animal Behaviour Science, n.32, p.325-335, 1992.

BOUCHARD, T.J.; LYKKEN, D.T.; MCGUE M. et al. Sources of human psychological differences: The Minnesota study of twins reared apart. Science, 250, p.223-228, 1990.

CURLEY JR., K.O.; NEUENDORFF, D.A.; LEWIS, A.W et al. Evaluation of temperament and stress physiology may be useful in breeding programs. In: Beef Cattle Research in Texas Publication, section Physiology. Disponível em: www.animalscience.tamv.edu/ansc/beef/bcrt/2004/curley> Acesso em: 20/3/2007.

DIAZ, J.MG. ; FISCHER, V. ; POLI, C.H.E.C. et al. Efeito da oferta de forragem, método de pastejo, dias de avaliação e raças no comportamento e temperamento de ovinos. Revista Brasileira de Zootecnia, 2010 (no prelo).
DICKSON, D.P.; BARR, G.R.; JOHNSON, L.P. et al. Social dominance and temperament of Holstein cows. Journal of Dairy Science, v.53, p.904-907, 1970.

FORDYCE, G.; GODARD, M.; SEIFERT, G.W. The measurement of temperament in cattle and effect of experience and genotype. Animal Production in Australian, n.14, p.329-332, 1982.

FORDYCE, G.; DODT, R.M.; WYTHES, J.R. Cattle temperaments in extensive beef herds in northern Queensland. 1. Factors affecting temperament. Australian Journal of Experimental Agriculture, v.28, n.6, p.683-687, 1988.

FRASER, A.F.; BROOM, D.M. Farm animal behaviour and welfare. London: CABI, 1997. 437p.

GRANDIN, T. Voluntary acceptance of restraint by sheep. Applied Animal Behavioral Science, v.23, p.257-261, 1989.

GRANDIN, T. Effect of genetics on handling and CO2 stunning of pigs. Meat Focus International, (with july 2008 updates) p.124-126, 1992. Disponível em: <http://www.grandin.com/ humane/meatfocus7-92.html>. Acesso em: 15/1/2007.

GRANDIN, T. Agitated wild behaviour is persistent over time in exotic crossbred cattle. Applied Behaviour Science, v.36, p.1-9, 1993

GRANDIN, T.; DEESING, D. Genetics and behavioural of domestic animals. San Diego: Academic Press, 1998. 378p.

GRANDIN, T. Livestock handling and transport. Wallingford: CABI Publishing, 2000. p.63-85.

HEMSWORT, P.H.; BARNET, J.L.; TREACY, D. et al. Applied Animal Behaviour Science, v.25, p.85-95, 1990.

HEMSWORTH, P.H.; BARNETT, J.L. The effects of aversively handling pigs, either individually or in groups, on their behaviour, growth and corticosteroids. Applied Animal Behaviour Science, v.30, p.61-72, 1991.

HIXON, D.L.; KESLER, D.K.; TROXEL, T.R. Reproductive hormone secretions and first service conception rate subsequent to ovulation control with Synchromate B. Therio, v.16, p. 219-229, 1981

LANIER, J.L.; GRANDIN, T.; GREEN, R.D. The relationnship between reaction to sudden, intermittent moviments and sounds and temperament. Journal of Animal Science, v.78, n.6 p.1467-1474, 2000.

LE NEINDRE, P.; TRILLAT, G.; SAPA, J. et al. Individual differences in docility in Limousin cattle. Journal of Animal Science, v.73, p.2249-2253, 1995

ROMEYER, A.; BOUISSOU, M.F. Assessment of fear reactions in domestic sheep and influence of breed and rearing conditions. Applied Animal Behaviour Science, v.34, p.93-119, 1992.

PARANHOS DA COSTA, M.J.R. Racionalização do manejo de bovinos de corte: comportamento, bem-estar e produtividade. In: CONGRESSO BRASILEIRO DE ZOOTECNIA, 11., 2001, Goiânia. Anais... Goiânia, 2001. p.118-124.

PIOVEZAN, U.; PARANHOS DA COSTA, M.J.R.; CROMBERG, V. et al. Cattle temperament: what are we measuring? In: CONGRESS OF THE INTERNATIONAL SOCIETY FO APPLIED ETHOLOGY, 32., 1998, Clermont-Ferrant. Proceedings... Clermont-Ferrant: International Society for Applied Ethology, 1998. v.32. p.211-211.

RECH, C.L.; RECH, J.L.; FISCHER, V. et al. Temperamento e comportamento materno-filial de ovinos das raças Corriedale e Ideal e sua relação com a sobrevivência dos cordeiros. Ciência Rural, v.38, n.5, p.1388-1393, 2008

SYME, L.A.; ELPHICK, G.R. Heart rate and the bahaviour of sheep in yards. Applied Animal Etology, v.9, p.31-35, 1983.

SHUPE, W.L. Transporting sheep to pastures and markets. St. Joseph: ASAE, 1978 (Technical Paper, 78-6008).

STERMER, R.A.; CAMP, T.H.; STEVENS, D.G. Feeder cattle stress during handling and transportation. American Society of Agricultural Engineers Technical Paper, n.81, p.6001, 1981.

STOBEL, D.P.; MOBERG, G.P. Repeated acute stress during follicular phase and luteinizing hormone surge in dairy heifers. Journal of Dairy Science, v.65, p.92-96, 1982. 
WHATLEY, J.; KILGOUR, R.; DALTON, D.C. Behaviour of hill country sheep breeds during farming routines. Palmerston North: New Zealand Society of Animal Production, 1974. p.34.
VOISINET, B.D.; GRANDIN, T.; TATUM, J.D. et al. Feedlot cattle with calm temperaments have higher average daily gains than cattle with excitable temperaments. Journal of Animal Science, v.75, p.892-896, 1997. 